\title{
Fundus Condition of a Patient with Treacher Collins Syndrome - Case Report
}

\author{
Ying Mao, Ya nan Zhang and Bin Li* \\ Beijing Institute of Ophthalmology, Beijing Tongren Eye Center, Beijing Tongren Hospital, Capital Medical University, Beijing Ophthalmology \& Visual Sciences \\ Key Laboratory, Beijing, China
}

Received: 䟱: December 19, 2018; Published: 制: January 03, 2019

*Corresponding author: Bin Li, MD, Beijing Institute of Ophthalmology, Beijing TongRen Hospital, Capital Medical University, 17 Hougou Lane, Chongnei Street, 100005, Beijing, China
Abstract
The case report presents a child with Treacher Collins syndrome whose ocular surface was abnormal and accompanied binocular amblyopia, but ophthalmic examinations revealed there were no abnormalities in the fundus surprisingly. This case suggested us that Treacher Collins syndrome may only cause ocluar surface malformations but has no effect on the fundus.
Keywords: Treacher Collins Syndrome; Fundus
Abbreviations: TCS: Treacher Collins Syndrome; MFD: Mandibulofacial Dysostosis; OCT: Optical Coherence Tomography

\section{Introduction}

Treacher Collins syndrome (TCS), also known as mandibulofacial dysostosis (MFD). TCS patients were almost autosomal dominant inheritance, the incidence rate was $1 / 50000,40 \%$ of patients had a family history of disease, while sporadic patients account $60 \%$. The clinical features of TCS patients were perceived easily, which can be identified according to facial features [1]. Patients of TCS had an abnormal craniofacial appearance, but the status of their intelligence and growth were normal, expect very few patients were stunted [2]. TCS patients can be briefly divided into five types:

a) Complete Type: Eyelid, maxillofacial bone and ear malformation

b) Incomplete Type: Eyelid l and maxillofacial bone deformity the ear shape was normal but with hearing impairment

c) Frustrated Type: Only eyelid involved

d) Unilateral Type: Head and face manifestations were only one side deformities, while the contralateral side were normal

e) Irregular type.

Above of all, most TCS patients had eye problems, but only the ocular surfaces were described. In this case, the fundus of TCS patient was evaluated by fundus image and OCT. The results showed that the fundus morphology of this TCS patient was normal. These results suggested that the visual organs development of TCS patients may not be affected, but only involved in ocular surface morphology.

\section{Case Presentation}

A 5-year-old boy with Treacher Collins Syndrome presented with a 1-month history of binocular blurred vision. The boy height 1.1 meters ( $3.6 \mathrm{ft}$ ), weight 36 kilograms, the intelligence is normal. There was no family history of genetic diseases, and the parental prenatal examination was generally normal. The mother is healthy during pregnancy, caesarean section after 38 weeks of pregnancy; new born hearing screening test found a poor hearing.

\section{On Physical Examination}

The development of the skull was almost normal,only the occipital posterior process, palpation examination seems showed that had a part defect of the orbital bone. Hair growth extends to the cheeks. The boy was born with hypoplasia of the zygomatic arches, mandible, and external ear canals. He had an underdevelopment of the lower jaw; Underdevelopment of the zygomatic bone gives the cheeks a sunken appearance. The external ear is malformed, and the ear's position is lower than normal, the tragus is in front of the normal position, and bilateral narrowing of the external ear canals. As a result of these abnormalities of ear, he had conductive hearing loss. The patient had used a bone-conduction hearing aid since early childhood, enabled him to appreciate the sound and mastered speech. His nose was wider than normal, and the septum was not 
deflected. The lips were thick, and the mouth was large. when he opened his mouth, we could see his high arch without cleft palate, the small mandible that may cause a poor occlusion of the teeth's, the dentitions were not neatly. There were no obvious deformities in the bones and spine of the limbs.

\section{Ophthalmic Examination}

Naked eye vision: right eye (OD) : 0.2 , left eye (OS): 0.2 . Corrected visual acuity: OD: $+2.00 \mathrm{DS} /+4.00 \mathrm{DC} \times 130^{\circ}=0.3$; OS: $+2.00 \mathrm{DS} /+4.00 \mathrm{DC} \times 65^{\circ}=0.4$. Intraocular pressure: $16 \mathrm{mmHgs}$ $(1 \mathrm{mmHg}=0.133 \mathrm{kPa})$ in the right eye and $13 \mathrm{mmHgs}$ in the left eye. The Palpebral fissues of the left and right eyes were in a "V" shape, tilting outward and downward. Both sides of the upper eyelids were extended to the temporal. Drooping of both upper eyelids, covering the edge of the pupil $2 \mathrm{~mm}$; The lower eyelids on both sides were turned outwards and incomplete closure, colobomata (notches) could be found in the right lower eyelid, partial absence of eyelashes on the bilateral lower lid (Figure 1). The conjunctiva of both eyes was congested, the corneas were clear, FL: spotted fluorescent staining on the corneal surface of both eyes, especially at the lower eyelid near the limbus. The depth of the anterior chambers of both eyes were normal, the aqueous humours were clear, pupils were round in which that the pupil diameters were about $4 \mathrm{~mm}$. The direct and indirect reflect of light were exists and the lens were clear. Both ocular movements of both eyes were normal.

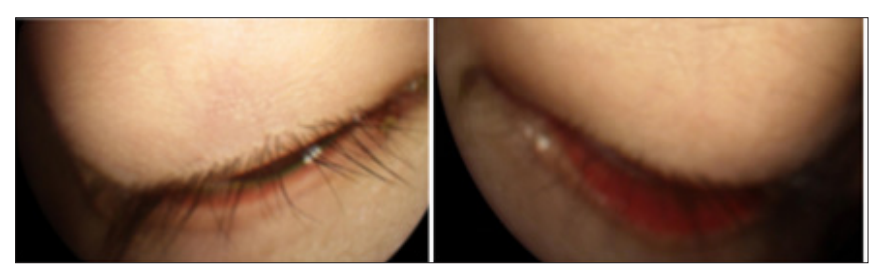

Figure 1: A: Shows the right eye; B: Shows the left eye.

\section{Fundus Examination}

Because the child is photophobic and poorly matched, the quality of the fundus image is not good. Fundus examination revealed no obvious abnormality (Figure 2). The posterior segment of the frequency domain OCT shows: There were no obvious abnormalities in the interlaminar structure of the retinal macular area, the papillary morphology and the thickness of the nerve fibre layer of both eyes (Figure 3). ultrasound B-scan: the right eye axis length is $21.9 \mathrm{~mm}$, and the left eye axis length is $21.70 \mathrm{~mm}$. Due to the poor coordination of the children that failed to check the X-ray and MRI examinations. Diagnosis: Treacher Collins syndrome:

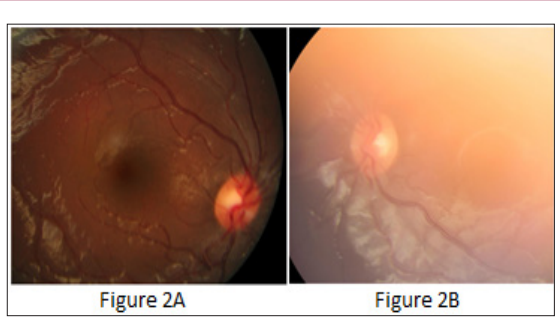

Figure 2: A: Shows the right eye; B: Shows the left eye.

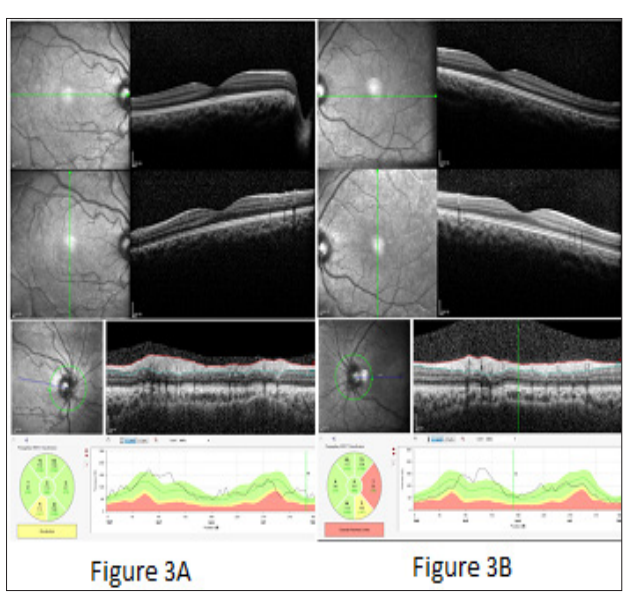

Figure 3: A: Shows the right eye; B: Shows the left eye.

a) Enlargement of palpebral fissue, eyelid ectropion, bilateral exposure keratitis, binocular ametropia

b) Maxillofacial dysplasia, mandibular shortness

c) External auricle dysplasia, Stenosis of external auditory meatus, hearing abnormality.

\section{Discussion}

The symptoms of most TCS patients had significant clinical heterogeneities, the causes of the differences were mostly considered to be the genetically heterogeneous. According to the gene mutations that classifies TCS patients in to three types: TCS-1 type (OMIM 154500) caused by TCOF1 gene mutation, TCS-2 type (OMIM 613717) caused by mutation of POLR1D gene and TCS3type (OMIM 248390) caused by mutation of POLR1C gene. The TCOF1 gene is located at 5q32-q33.1, which is the most common causative gene that present in $81 \%$ to $93 \%$ of cases. Because this gene regulated a specific stage in the process of ribosome formation, its mutation lead to change the treacle protein, which affected the formation of bone and other tissues in the early stage of embryo development process. Therefore, this mutation may lead to the diversity of clinical manifestations of TCS patients [3,4]. It was important to investigate the parents to determine whether the affected genes were present, in this case, the risk of having another affected child was $50 \%$. On the contrary, If the parents did not have the affected gene, the recurrence risk appears to be low.

Therefore, providing genetic counselling to families, determining genetic risk, and prenatal diagnosis were extremely helpful [5]. The patient's condition was relatively stable, and the symptoms did not progress with age. Ophthalmological examination showed no obvious abnormality in the intraocular morphology. In addition, the binocular deprivation amblyopia was caused by mild drooping of the double upper eyelids, covering the pupillary margins by about $2 \mathrm{~mm}$, which affects the visual development of the child. The patient was seeking the treatments of binocular blurred vision, according to the principle of individualized treatment, Ophthalmic treatment plans were mainly performs amblyopia training that to correct the visual acuity of the child, and performs ocular surface plastic 
surgery to improve the mild drooping of the upper eyelid and the incomplete closure of the double eyes, which further improves the normal structure and developed high-level visual functions. Protects the corneal epithelium from the secondary infections.

Due to the multiple lesions in patients, it is usually needing to unite multidisciplinary departments, like the department of plastic surgery, department of otorhinolaryngology head and neck surgery, department of Stomatology, etc. to carry out staged reconstruction surgeries that to promote the development of speech and hearing [6]. The both eyes' lower eyelids were turned outwards and incomplete closure (right eye fissure was approximate $1 \mathrm{~mm}$, left eye fissure was approximate $1.5 \mathrm{~mm}$ ), partial absence of eyelashes on the bilateral lower lid. The binocular optic disc was round, the border was clear, and the colour were reddish, $C / D=0.4$. The shape of retinal vascular were generally normal, $A: V=2: 3$, the arteries and veins tube diameter of fundus were uniform, due to poor coordination of the child, the macular area is unclear. No abnormal reflections were observed in the binocular optic disc and the macular area. In these two eyes, the retinal structures of the macular area were intact, and the surface of the retinal nerve fibre layers were continuous and smooth, only the thickness of the nerve fibre layer near temporal side of left eye was thin, and no other obvious abnormalities were found. The retinal pigment epithelial layers were continuous, smooth, and closely connected to the choroidal capillary layers.

ISSN: 2574-1241

DOI: 10.26717/BJSTR.2019.12.002303

Bin Li. Biomed J Sci \& Tech Res

This work is licensed under Creative Commons Attribution 4.0 License

Submission Link: https://biomedres.us/submit-manuscript.php

\section{Funding/Support}

Beijing Natural Science Foundation (grant No. 7162036, 2016) and Beijing Institute of Ophthalmology-led project of key discipline (grant No. 201505, 2015) provided financial support. The sponsors or funding organizations had no role in the design or conduct of this research.

\section{References}

1. Trainor P, Dixon J, Mj (2009) Treacher Collins syndrome: etiology, pathogenesis and prevention. Eur J Hum Genet 17(3): 275-283.

2. Kadakia S, Helman S N, Badhey A K, Saman M, Ducic Y (2014) Treacher Collins Syndrome: The genetics of a craniofacial disease. Int J Pediatr Otorhinolaryngol 78(6): 893-898.

3. Gladwin AJ, Bonner CA, Dixon J, Sara J Edwards, Kathryn Koprivnikar, et al. (1996) Positional cloning of a gene involved in the pathogenesis of Treacher Collins syndrome. Nature Genet 12(2): 130-136.

4. Matsumoto N, Kaneko M, Watanabe N, Itaoka M, Seki Y, et al. (2018) Treacher Collins syndrome 3 (TCS3)-associated POLR1C mutants are localized in the lysosome and inhibits chondrogenic differentiation. Biochem Biophys Res Commun 499(1): 78-85.

5. Sakai D, Trainor PAJ (2009) Treacher Collins syndrome: Unmasking the role of Tcof1/treacle. Int J Biochem Cell Biol 41(6): 1229-1232.

6. Cobb ARM, Green B, Gill D, Ayliffe P, Lloyd TW, et al. (2014) The surgical management of Treacher Collins syndrome. Br J Oral Maxillofac Surg 52(7): 581-589.

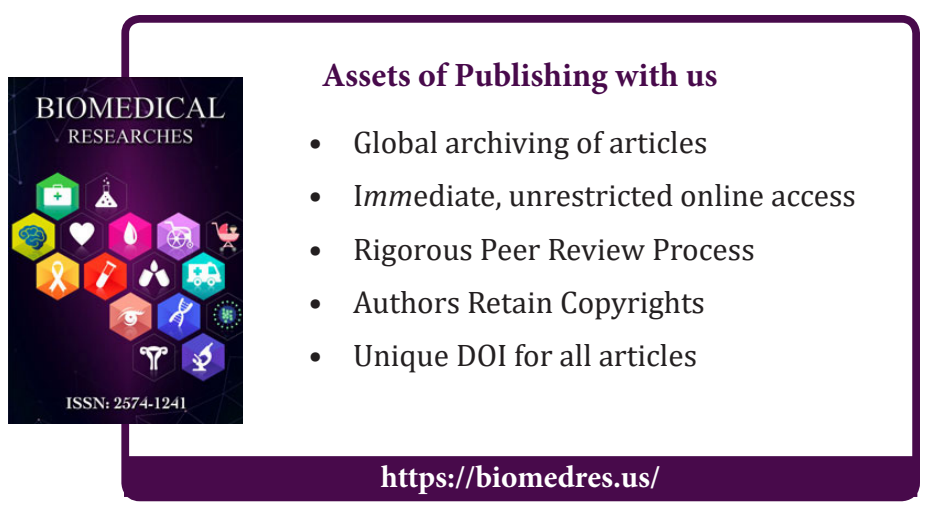

Cite this article: Ying Mao, Ya nan Zhang,Bin Li. Fundus Condition of a Patient with Treacher Collins Syndrome - Case Report. Biomed J Sci \& 